\title{
Patient's body composition can significantly affect RF power deposition in the tissue around DBS implants: ramifications for lead management strategies and MRI field-shaping techniques
}

21 August 2020

REVISED

5 November 2020

ACCEPTED FOR PUBLICATION

25 November 2020

PUBLISHED

13 January 2021

\author{
Bhumi Bhusal $^{1}$ (D) , Boris Keil ${ }^{2}$, Joshua Rosenow ${ }^{3}$, Ehsan Kazemivalipour ${ }^{4}$ and Laleh Golestanirad ${ }^{1,5}(\mathbb{D}$ \\ 1 Department of Radiology, Northwestern University, Chicago, IL, United States of America \\ 2 Institute of Medical Physics and Radiation Protection, Mittelhessen University of Applied Sciences, D-35390 Giessen, Germany \\ 3 Department of Neurosurgery, Northwestern University, Chicago, IL, United States of America \\ 4 Department of Electrical and Electronics Engineering, Bilkent University, Ankara, Turkey \\ 5 Department of Biomedical Engineering, Northwestern University, Evanston, IL, United States of America \\ E-mail:Laleh.rad1@northwestern.edu
}

Keywords: body models, magnetic resonance imaging (MRI), deep brain stimulation (DB)S, finite element modeling, RF heating, safety Supplementary material for this article is available online

\begin{abstract}
Patients with active implants such as deep brain stimulation (DBS) devices have limited access to magnetic resonance imaging (MRI) due to risks associated with RF heating of implants in MRI environment. With an aging population and increased prevalence of neurodegenerative disease, the indication for MRI exams in patients with such implants increases as well. In response to this growing need, many groups have investigated strategies to mitigate RF heating of DBS implants during MRI. These efforts fall into two main categories: MRI field-shaping methods, where the electric field of the MRI RF coil is modified to reduce the interaction with implanted leads, and lead management techniques where surgical modifications in the trajectory reduces the coupling with RF fields. Studies that characterize these techniques, however, have relied either on simulations with homogenous body models, or experiments with box-shaped single-material phantoms. It is well established, however, that the shape and heterogeneity of human body affects the distribution of RF electric fields, which by proxy, alters the heating of an implant inside the body. In this contribution, we applied numerical simulations and phantom experiments to examine the degree to which variations in patient's body composition affects RF power deposition. We then assessed effectiveness of RF-heating mitigation strategies under variant patient body compositions. Our results demonstrated that patient's body composition substantially alters RF power deposition in the tissue around implanted leads. However, both techniques based on MRI field-shaping and DBS lead management performed well under variant body types.
\end{abstract}

\section{Introduction}

More than 12 million people in the united states are presently carrying a form of conductive medical implant such as cardiac pacemakers or neuromodulation devices (Philips). It is estimated that $50 \%-75 \%$ of these patients will need magnetic resonance imaging (MRI) exams during their lifetime (Kalin and Stanton 2005), with many patients requiring repeated examination (Naehle et al 2009). The major risk of performing MRI on patients with electronic implants is due to the radiofrequency (RF) heating of the tissue surrounding the implant's tip, a phenomenon commonly known as the antenna effect (Yeung et al 2001, Park et al 2007, Bhusal et al 2018). In cases such as deep brain stimulation (DBS) devices where leads are implanted in sensitive organs as the brain, highly restrictive guidelines are in place to safeguard patients, limiting MRI accessibility. DBS manufacturers for instance, have limited use of MRI to $1.5 \mathrm{~T}$ horizontal scanners, and only with pulse sequences that generate whole-head specific absorption rate (SAR) of RF energy less than $0.1 \mathrm{~W} \mathrm{~kg}^{-1}$ (30 times below the

(C) 2021 Institute of Physics and Engineering in Medicine 
FDA limit for scanning in the absence of implants) or the magnetic field $\mathrm{B}_{1}^{+} \mathrm{rms} \leqslant 2 \mu \mathrm{T}$ (Medtronic 2015, St Jude Medical 2018, Boston Scientific 2019). Complying with these guidelines has proven to be difficult. For example, a typical stroke MRI protocol consists of T2/FLAIR, perfusion images and MR angiography (Wintermark et al 2008). These sequences have $S A R$ and $B_{1}$ levels well beyond what is allowed in patients with DBS implants. The situation is even more problematic within the context of musculoskeletal (MSK) imaging, for which the prescribed sequences have SAR levels already approaching the FDA limit for scanning even in the absence of implants. Among patients with movement disorders who are prone to falls and joint injuries, MSK MRI is often indicated, leaving those with DBS implants unable to receive the standard of care.

Currently 3T MRI is contraindicated for majority of DBS patients. There are, however, strong incentives to make 3 T MRI accessible for DBS imaging as it confers a much better contrast-to-noise ratio, allowing to delineate small structures surrounding DBS targets which is crucial for electrode localization (Cheng et al 2014). Unsurprisingly, recent years have witnessed considerable efforts to reduce RF heating of DBS implants during MRI, both through MRI hardware modification, and by modifying the implanted lead's design, material, and trajectory. From MRI hardware perspective, promising studies have shown the possibility of applying parallel transmit technology (Eryaman et al 2014, McElcheran et al 2017,2019, Guerin et al 2020), and reconfigurable MRI coils (Golestanirad et al 2017b, 2017c, 2019b, Kazemivalipour et al 2019) to shape the electric field of the MRI transmit coil in each individual patient such that field interactions with DBS leads is minimized. Other efforts have focused on modifying the implant's design and material (Serano et al 2015, McCabe and Scott 2017, Golestanirad et al 2019a) or its trajectory inside the body (Golestanirad et al 2017a, 2019c) to reduce the coupling of implanted leads with MRI electric fields. However, these studies have either relied on simulations in homogenous body models, or experiments with box-shaped single-material phantoms. This is a limitation, as the distribution of MRI electric fields inside a sample can be substantially altered by changes in local electric properties of the sample (Alon et al 2016), which consequently affects RF heating of a conductive implant (Fujimoto et al 2018). The problem is particularly important in the context of implant heating, as a recent study with a commercial DBS device implanted in a multi-material anthropomorphic phantom reported a substantial increase in RF heating in phantoms with subcutaneous fat compared to phantoms with no fatty tissue (Bhusal et al 2020). This raises the concern as whether or not the promising strategies previously introduced by reconfigurable MRI coil technology and surgical lead management are applicable in patients with diverse body characteristics.

In this work, we present results of numerical simulations to investigate major concerns regarding applicability of RF coil modifications and DBS lead management strategies to reduce RF power deposition in the tissue around the DBS contacts across patients with different body types. Specifically, we aim to address three open questions that help advancing development of such techniques:

\subsection{Can numerical simulations reliably predict alteration of RF power deposition due to variations in patient's body composition?}

As experiments to establish safety of implants in MRI environment are expensive and time consuming (Shrivastava et al 2010, 2012), there are increasing efforts towards application of numerical simulations (Lattanzi et al 2009, Golestanirad et al 2012a, 2019d). A recent phantom study reported substantial variation in RF heating of DBS implants due to variation in phantom composition (Bhusal et al 2020). We performed numerical simulations to investigate whether these experimental observations could be replicated in silico, and whether more realistic body models with and without local fat around the implanted pulse generator (IPG) would show the same trend.

\subsection{Are DBS lead management strategies that aim to reduce MRI heating effective in patients with diverse body types?}

Introducing loops in the extracranial trajectory of DBS leads can substantially reduce RF heating during MRI as shown in simulations with homogenous head models and experiments with single-material phantoms (Baker et al 2005, Golestanirad et al 2017a). A suggested mechanism for this SAR reduction is that the voltage induced in the lead by the incident electric field of MRI scanner is canceled out along the opposite sides of the loop, leading to a smaller induced current in the lead (Golestanirad et al 2019c). Consequently, looping DBS leads at the surgical burrhole or on the temporal bone has been suggested and applied in clinic (Baker et al 2005). However, as presence of fat alters the distribution of the electric field and subsequently changes the RF heating, it is important to examine whether or not lead management strategies will be effective across different body characteristics. In this work, we report simulation results of clinically-relevant DBS lead configurations including those with extracranial loops, in body models with and without subcutaneous fat and examine the effectiveness of lead management techniques across different body types. 


\subsection{Are MRI hardware modification techniques based on field-shaping effective in patients with diverse body types?}

In the context of MRI hardware modification for DBS imaging, reconfigurable patient-adjustable (RPA) coils have shown promise to reduce RF heating of DBS implants on a patient-specific basis (Golestanirad et al 2017b, Kazemivalipour et al 2019). The technique works by rotating a linearly-polarized (LP) birdcage transmit coil, which has a slab-like region of zero electric field, around patients head, such that implanted DBS leads are contained within the zero electric field region of the coil. To date, all studies that assessed feasibility and performance of RPA coils were performed with homogenous head models and homogenous phantoms. Here, we performed finite element simulations to quantify, for the first time, the degree to which SAR- reduction performance of an MRI reconfigurable coil is dependent on patient's body characteristics.

This work aims to set the ground for further development and dissemination of techniques that reduce RF heating of DBS implants through MRI hardware modification and surgical lead management strategies. If successful, clinical implementation of such techniques would impact patient's care in a large scale.

\section{The effect of subcutaneous fat on RF heating of DBS devices during MRI with body coils}

In order to assess how the presense of body fat affects the RF heating of DBS implants, we performed experiments with a commercially available DBS device (Medtronic Inc., Minneapolis, MN) implanted in an anthropomorphic phantom with and without added fat, and investigated whether a similar pattern of RF heating can be predicted by numerical simulations. Below is the description of experimental setup and simulations.

\subsection{Experimental setup}

An anthropomorphic phantom consisting of a human-shaped torso and skull was designed and fabricated along with grids and supporting structures that allowed DBS device to be positioned in a manner similar to the clinical practice (figure 1). The skull was filled with saline-doped agar gel $\left(\sigma=0.40 \mathrm{~S} \mathrm{~m}^{-1}\right.$ and $\left.\varepsilon_{r}=78\right)$ and inserted into the body of the phantom which was then filled with $18 \mathrm{lof}$ saline solution $\left(\sigma=0.50 \mathrm{~S} \mathrm{~m}^{-1}\right.$ and $\left.\varepsilon_{r}=78\right)$. The saline solution fully immersed the lead-extension trajectory and the IPG was about $3 \mathrm{~cm}$ beneath the surface of saline. A Medtronic DBS device (Medtronic Inc., Minneapolis, MN) with a $40 \mathrm{~cm}$ lead (model 3387), $60 \mathrm{~cm}$ extension (model 3708660) and an implantable pulse generator (IPG) (Activa PC-37601) was implanted in the phantom. MR compatible flouroptic temperature probes (OSENSA, BC, Canada, resolution $0.01^{\circ} \mathrm{C}$ ) were secured to the lead contacts (contact- 0 and contact- 2 ) and the lead-probe system was inserted into the skull through a $5 \mathrm{~mm}$ hole drilled on the surface. The phantom was positioned in the MRI scanner such that its head was at the iso-center of the magnet, mimicking RF exposure during brain imaging. RF heating was measured for four extracranial lead trajectories with extracranial loops at different positions as depicted in figure 1. All four trajectories followed the same path on the skull and towards the IPG, except for the variation in the loop position; therefore, any change in the heating was primarily due to the variation in the loop position. This was assured by drawing trajectory path along the skull and using a fixed support stand on the path towards the IPG. Tajectory A consisting of concentric loops at the surgical burrhole has been suggested in previous works to reduce RF heating (Baker et al 2005, Golestanirad et al 2017a). Trajectories B, C and D are representative of other relevant clinical scenarios as observed from post-operative CT images of DBS patients (Golestanirad et al 2019c).

RF heating measurements were performed at a Siemens $3 \mathrm{~T}$ Prisma scanner (Siemens Healthineers, Erlangen, Germany), using body transmit coil and a 20-channel receive head coil. A $\mathrm{T}_{1}$-weighted turbo spin echo sequence with $\mathrm{TE}=7.5 \mathrm{~ms}, \mathrm{TR}=1450 \mathrm{~ms}$, flip angle $=150^{\circ}, \mathrm{B}_{1}^{+} \mathrm{rms}=2.8 \mu \mathrm{T}$ and acquisition time of $7 \mathrm{~min}$ $31 \mathrm{~s}$ was used during RF exposure. Experiments were repeated by replacing $7 \mathrm{l}$ of saline by the same amount of vegetable oil which created a $3 \mathrm{~cm}$ layer of fat on top of the saline solution, mimicking presence of subcutaneous fat. The amount of oil was chosen such that the upper surface of the IPG as well as the extension trajectory around the IPG could be covered in oil.

\subsection{Simulations}

\subsubsection{Models mimicking phantom experiments}

To investigate how variations in the distribution of electromagnetic fields due to presence of fat contribute to the changes observed in the RF heating we replicated the experimental setup in finite element simulations. Simulations were implemented in ANSYS Electronic Desktop 2019 (ANSYS, Canonsburg, Pennsylvania, USA), following a combined finite element-circuit analysis approach (Kozlov and Turner 2009). The simulated phantom consisted of models of the brain, skull, and body similar to the experimental phantom, with all tissues assigned to the same dielectric properties as measured in the experiments and reported above. Four DBS lead trajectories mimicking experimental setup were created as illustrated in figure 1. For this, we used 

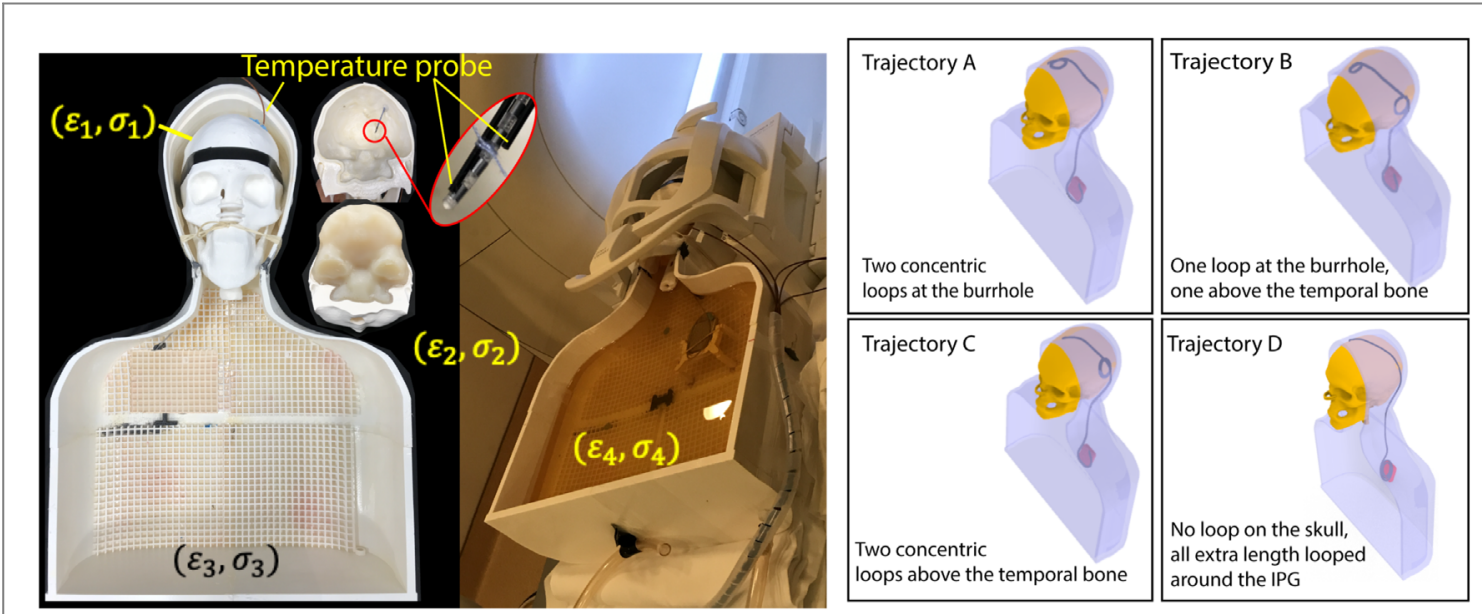

Figure 1. Left: experimental setup showing the anthropomorphic phantom. The skull structure was $3 \mathrm{D}$ printed in plastic with low conductivity and permittivity $\left(\sigma_{1}=0 \mathrm{~S} \mathrm{~m}^{-1}\right.$ and $\left.\varepsilon_{r 1}=3.5\right)$ similar to bone and was filled with brain mimicking gel $\left(\sigma_{2}=0.40 \mathrm{~S} \mathrm{~m}^{-1}\right.$ and $\left.\varepsilon_{r 2}=78\right)$. The torso was filled with saline $\left(\sigma_{3}=0.50 \mathrm{~S} \mathrm{~m}^{-1}\right.$ and $\left.\varepsilon_{r 3}=78\right)$ and oil $\left(\sigma_{4}=0 \mathrm{~S} \mathrm{~m}^{-1}\right.$ and $\left.\varepsilon_{r 4}=3\right)$. The DBS device was positioned inside the phantom similar to clinical practice. Right: different configurations of extracranial lead and extension trajectories, mimicking different surgical strategies.

computer-aided design tool (Rhino 6.0, Robert McNeal \& Associates, Seattle, WI) to draw trajectory curves along the skull, resembling the path in experiments. We then added the loops along the path at different locations same as in experiment to create the four different trajectories. The curves were imported into ANSYS HFSS and piped to create the model of the lead and insulation. MRI RF coil was modeled as a 16-rug high-pass birdcage (67 cm length $61 \mathrm{~cm}$ diameter), tuned at $127 \mathrm{MHz}(3 \mathrm{~T})$, and driven in quadrature through two signal sources placed at the end ring on patient's head side. Details of the coil geometry and tuning capacitors are given elsewhere (Nguyen et al 2020). The input power of the coil was adjusted such that it produced a mean $\mathrm{B}_{1}^{+}=2.8 \mu \mathrm{T}$ on a circular plane at its iso-center, similar to the $\mathrm{B}_{1}^{+}$reported by the scanner during $\mathrm{RF}$ exposure experiments. The maximum of $1 \mathrm{~g}$-averaged SAR was calculated around the tip of DBS leads for each scenario to be compared with experimental results. All simulations were performed twice, once with a phantom without the fat, and a second time with a layer of fat covering the surface of phantom's body similar to the experimental setup.

\subsubsection{Realistic body model}

It is important to note that in reality, overweight patients have increased amount of local subcutaneous fat mostly in the chest area surrounding the IPG. This is different from our experimental setup, where the oil layer fully covered the surface of phantom's body. To examine if a similar trend in variation of RF heating was present in a case that represented human body more realistically, we also performed simulations with a human-shaped heterogenous body model consisting of average tissue $\left(\sigma=0.47 \mathrm{~S} \mathrm{~m}^{-1}\right.$ and $\left.\varepsilon_{r}=78\right)$, Bone $\left(\sigma=0.07 \mathrm{~S} \mathrm{~m}^{-1}\right.$ and $\left.\varepsilon_{r}=15\right)$, brain tissue $\left(\sigma=0.40 \mathrm{~S} \mathrm{~m}^{-1}\right.$ and $\left.\varepsilon_{r}=78\right)$, muscle tissue $\left(\sigma=0.70 \mathrm{~S} \mathrm{~m}^{-1}\right.$ and $\left.\varepsilon_{r}=64\right)$, lungs $\left(\sigma=0.31 \mathrm{~S} \mathrm{~m}^{-1}\right.$ and $\left.\varepsilon_{r}=30\right)$, cartilage $\left(\sigma=0.48 \mathrm{~S} \mathrm{~m}^{-1}\right.$ and $\left.\varepsilon_{r}=53\right)$, internal air $\left(\sigma=0 \mathrm{~S} \mathrm{~m}^{-1}\right.$ and $\left.\varepsilon_{r}=1\right)$, and with and without a block of local fat $\left(\sigma=0.04 \mathrm{~S} \mathrm{~m}^{-1}\right.$ and $\left.\varepsilon_{r}=6\right)$ covering the upper face of the IPG and initial segments of the extension as demonstrated in figure 2(B). The fat block was morphed to fit the shape of pectoral region and had maximum thickness of $2 \mathrm{~cm}$.

\subsubsection{Numerical convergence}

The initial mesh was set such that maximum element size was $<2 \mathrm{~mm}$ for the lead, $<4 \mathrm{~mm}$ for the insulation, $<5 \mathrm{~mm}$ for IPG, $<10 \mathrm{~mm}$ for the coil, $<20 \mathrm{~mm}$ for brain, body, and coil shield, and $<10 \mathrm{~mm}$ for local fat and skull. ANSYS HFSS follows an adaptive mesh scheme with successive refinement of an initial mesh between iterative passes. At each adaptive pass, scattering parameters ( $S$-parameters) are evaluated at each port, and compared to the previous pass. The change in magnitude of the $S$-parameters between two consecutive passes is called 'delta $S$ '. The maximum delta $S$ is defined as $\operatorname{Max}_{i j}\left|S_{i j}^{N}-S_{i j}^{N-1}\right|$, where $i$ and $j$ cover all ports and $N$ represents the number of iterative pass. Simulations were considered to be converged when the maximum delta $S$ fell below a set threshold of 0.02 . All simulations converged with 2-4 adaptive passes. The convergence of absorbed RF power density was verified by measuring local SAR (calculated as $\frac{\sigma|\boldsymbol{E}|^{2}}{\rho}$ ) as well as maximum $1 \mathrm{~g}$ averaged SAR for trajectory $\mathrm{C}$ with different convergence thresholds as shown in table 2 . Both local as well as $1 \mathrm{~g}$ averaged SAR changed by less than $1 \%$. Total time taken for each simulation was about two and half hours on a 




(A)

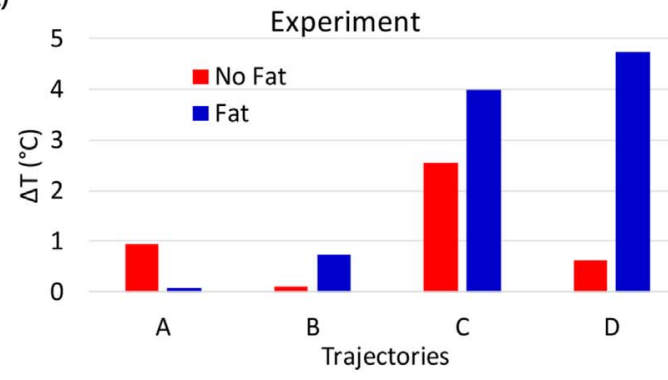

(B) Simulation with the phantom mimicking the experimental setup

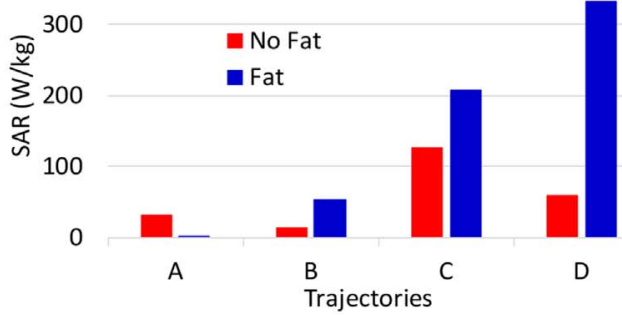

Figure 3. (A) Plot of experimentally measured temperature rise at DBS lead electrodes for lead trajectories A-D (as shown in figure 1) in phantoms with and without subcutaneous fat. (B) The maximum of 1 -averaged SAR at the tip of the simulated DBS lead in a phantom that mimicked the experimental setup.

Table 1. Mesh statistics for a simulation with experiment mimicking phantom with trajectory D and no. fat tissue.

\begin{tabular}{lcccc}
\hline & & $\begin{array}{c}\text { Min. } \\
\text { edge } \\
\text { length } \\
(\mathrm{mm})\end{array}$ & $\begin{array}{c}\text { Max. } \\
\text { edge } \\
\text { length } \\
(\mathrm{mm})\end{array}$ & $\begin{array}{c}\text { Rms } \\
\text { edge } \\
\text { length } \\
(\mathrm{mm})\end{array}$ \\
\hline Parts & tetrahedrons & 0.06 & 1.99 & 0.82 \\
Insulation & 84827 & 0.06 & 2.15 & 1.05 \\
Brain & 30716 & 0.11 & 27.05 & 11 \\
Skull & 79414 & 0.2 & 12.2 & 7.63 \\
SAR box & 41704 & 0.04 & 2.41 & 1.57 \\
Body & 144681 & 0.1 & 25.17 & 14.47 \\
\hline
\end{tabular}

DELL server with 1.5 TB memory and 2_Xenon(R) Gold 6140 CPUs each having 32 processing cores. Table 1 shows mesh statistics for a typical simulation. 

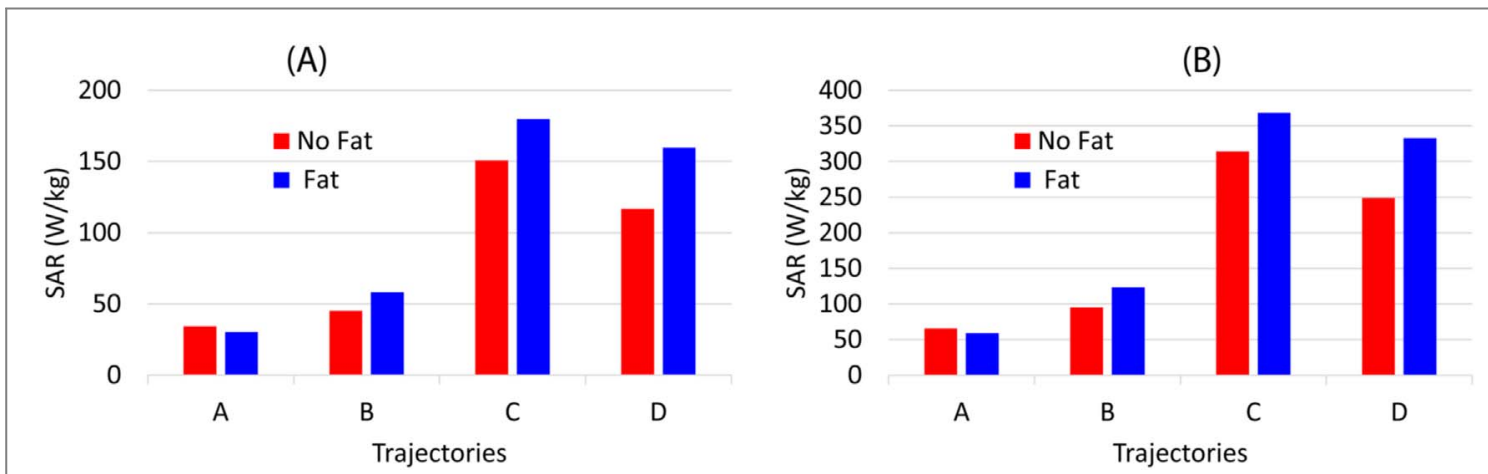

Figure 4. The maximum of 1g-averaged SAR at the tip of the simulated DBS lead in a realistic body model with and without local fat around the IPG. (A) SAR values for the input power adjusted to produce $\mathrm{B}_{1}^{+}$of $2.8 \mu \mathrm{T}$. (B) SAR values for the input power adjusted to produce whole head SAR of $3.2 \mathrm{~W} \mathrm{~kg}^{-1}$.

Table 2. Local and $1 \mathrm{~g}$ averaged MaxSAR values for trajectory $\mathrm{C}$ without fat, with different convergence thresholds.

\begin{tabular}{lccc}
\hline $\begin{array}{l}\text { Convergence criteria } \\
\left(\operatorname{Max} \_\Delta S\right)\end{array}$ & $\begin{array}{c}\text { No. of adaptive } \\
\text { passes completed }\end{array}$ & $\begin{array}{c}\text { MaxSAR1g } \\
\left(\mathrm{W} \mathrm{kg}^{-1}\right)\end{array}$ & $\begin{array}{c}\text { MaxSAR_local } \\
\left(\mathrm{W} \mathrm{kg}^{-1}\right)\end{array}$ \\
\hline 0.02 & 2 & 76.86 & $1.54 \mathrm{E}+05$ \\
0.007 & 3 & 77.31 & $1.55 \mathrm{E}+05$ \\
0.001 & 5 & 76.13 & $1.52 \mathrm{E}+05$
\end{tabular}

\subsubsection{Simulation versus experimental results}

Figure 3(A) shows the temperature rise $\Delta T$ measured at the tip of the DBS lead during RF exposure experiments with different lead trajectories of figure 1 in phantoms with and without subcutaneous fat. Figure 3(B) gives the numerical results for the maximum $1 \mathrm{gSAR}$ in simulations that mimicked the experimental phantom setup. As it can be observed, the effect of variation in phantom composition and lead trajectories on $\Delta T$ is well reflected in SAR simulations. Specifically, for the worst-case heating scenario (trajectory D), the presence of fat increased the measured $\Delta T$ by 7 -fold (from $0.61^{\circ} \mathrm{C}$ to $4.70^{\circ} \mathrm{C}$ ) and the calculated 1 gSAR by 6 -fold (from 29.7 to 169.4 $\mathrm{W} \mathrm{kg}^{-1}$ ).

Simulations with the realistic body model with and without local fat around the IPG predicted a similar trend in variation of RF heating due to presence of fat (figure 4). Specifically, for lead trajectories with highest heating (trajectories D and C) the addition of local fat around the IPG increased the $1 \mathrm{gSAR}$ at the electrode tip by $37 \%$, and $19 \%$ respectively, predicting the same trend observed in the experiments.

\subsubsection{Why presence of fat changes RF heating? Insights from RF field distribution}

RF heating of an implant is known to be highly affected by the background electric field of the MRI scanner (Nordbeck et al 2008). To assess how the distribution of the background electric field was varied due to changes in the phantom composition, we performed simulations in phantoms with and without fat, in the absence of the implant. Figure 5(A) shows simulated maps of the magnitude of incident electric field on three coronal planes in the phantoms at $17 \mathrm{~mm}$ below, $3 \mathrm{~mm}$ above, and $23 \mathrm{~mm}$ above the fat-saline interface (the implant is shown only for visualization and was not included in simulations). The distribution of $\mathrm{B}_{1}^{+}$field is also given on the plane that was $3 \mathrm{~mm}$ above the fat-saline interface (plane 2) in figure 5(B). As it can be observed, the electric field magnitude was substantially higher on planes that pass through the fat layer, even though the $\mathrm{B}_{1}^{+}$field remained relatively unchanged. Consequently, lead trajectories that pass through fatty tissue will be exposed to a higher electric field resulting in higher induced currents, even though they experience the same $\mathrm{B}_{1}^{+}$magnitude. This can be better appreciated from figure 5(D) where the peak value of the tangential component of the incident electric field, $E_{\tan }$, is shown along the lead trajectory D. On average, the magnitude of $E_{\tan }$ along the last $40 \mathrm{~cm}$ of the extension (the portion starting from the IPG) was 2.5 times higher in the phantom with additional fat than in phantom with only saline. Such increased incident electric field can lead to increased induced current along the DBS lead if the induced currents from this segment add up in phase to the currents from other segments, resulting in increased RF heating, as theoretically predicted by the concept of lead transfer function (Park et al 2007, Feng et al 2014, Nyenhuis et al 2015). Additionally, the presence of fatty tissue (low permittivity) can alter the resonance length of current along the leads which may change the RF heating at lead contacts. 




Figure 5. (A) Simulated maps of electric field (magnitude) distribution on three coronal planes positioned below and above fat-saline interface. Simulations are performed in phantoms with and without fat (where the properties of fat layer were changed to that of saline) in the absence of DBS implant. The implant is shown only for visualization. (B) Plot of $\mathrm{B}_{1}^{+}$on plane 2 in phantoms with and without fat. (D) Peak value of the tangential component of electric field along the length of the lead trajectory $\mathrm{D}$, in phantoms with and without fat.

\section{The effect of subcutaneous fat on the RF heating of DBS devices during MRI with head coils: application to the RPA coil technology}

The RPA coil technology has been recently introduced to reduce RF heating of DBS implants by rotating a linearly polarized birdcage transmit coil around patient's head such that the implant could be contained within the low electric field region of the coil (Golestanirad et al 2017b, 2019b, Kazemivalipour et al 2019). This reduces induced electric currents on the leads, which in turn reduces RF heating. To date, simulation studies and experimental measurements that characterized the performance of RPA technology have only used homogenous head or body models. Here we investigated the performance of a $3 \mathrm{~T}$ RPA coil in reducing RF heating of fully implanted DBS systems, and examined if increased amount of subcutaneous fat around the IPG would affect the SAR-reduction efficiency of the coil.

\subsection{Simulations}

We performed finite element simulations with a model of a shielded high-pass birdcage head transmit coil (16rung, $23.6 \mathrm{~cm}$ length, $30.6 \mathrm{~cm}$ diameter), tuned to $127 \mathrm{MHz}(3 \mathrm{~T}$ ), and driven in linear mode by a single sinusoidal source placed at one of the end rings. The coil was rotated around the head of the heterogeneous body model of figure 2 with $11.25^{\circ}$ increments to cover a full circle (figure 5). The maximum of 1 g-averaged SAR was calculated around the tip of the leads for trajectories A-D implanted in body models with and without local fat around the IPG. Simulations were repeated by replacing the linearly polarized birdcage head coil with the birdcage body coil of section 2 driven in quadrature mode (circular polarization) for calculation of SAR reduction efficiency (SRE). For all simulations, the input power of the body and the RPA coils were adjusted to produce mean $B_{1}^{+}=2 \mu \mathrm{T}$ on an axial plane passing through center of the coil. The maximum SRE of the RPA coil was quantified as:

$$
\mathrm{SRE}_{\text {max }}=100 \times \frac{\left(\mathrm{SAR}_{\mathrm{CP}}-\operatorname{SAR}_{\mathrm{LP}}\left(\theta_{\mathrm{opt}}\right)\right)}{\operatorname{SAR}_{\mathrm{CP}}},
$$

where $\mathrm{SAR}_{\mathrm{CP}}$ is the maximum of $1 \mathrm{~g}$-averaged SAR around the DBS lead generated by the CP body coil, $\theta_{\mathrm{opt}}$ is the optimum angle of the RPA coil that maximally reduces the $\operatorname{SAR}$, and $\operatorname{SAR}_{\mathrm{LP}}\left(\theta_{\mathrm{opt}}\right)$ is the maximum of $1 \mathrm{~g}$-averaged SAR when the RPA coil is positioned at its optimal angle. 


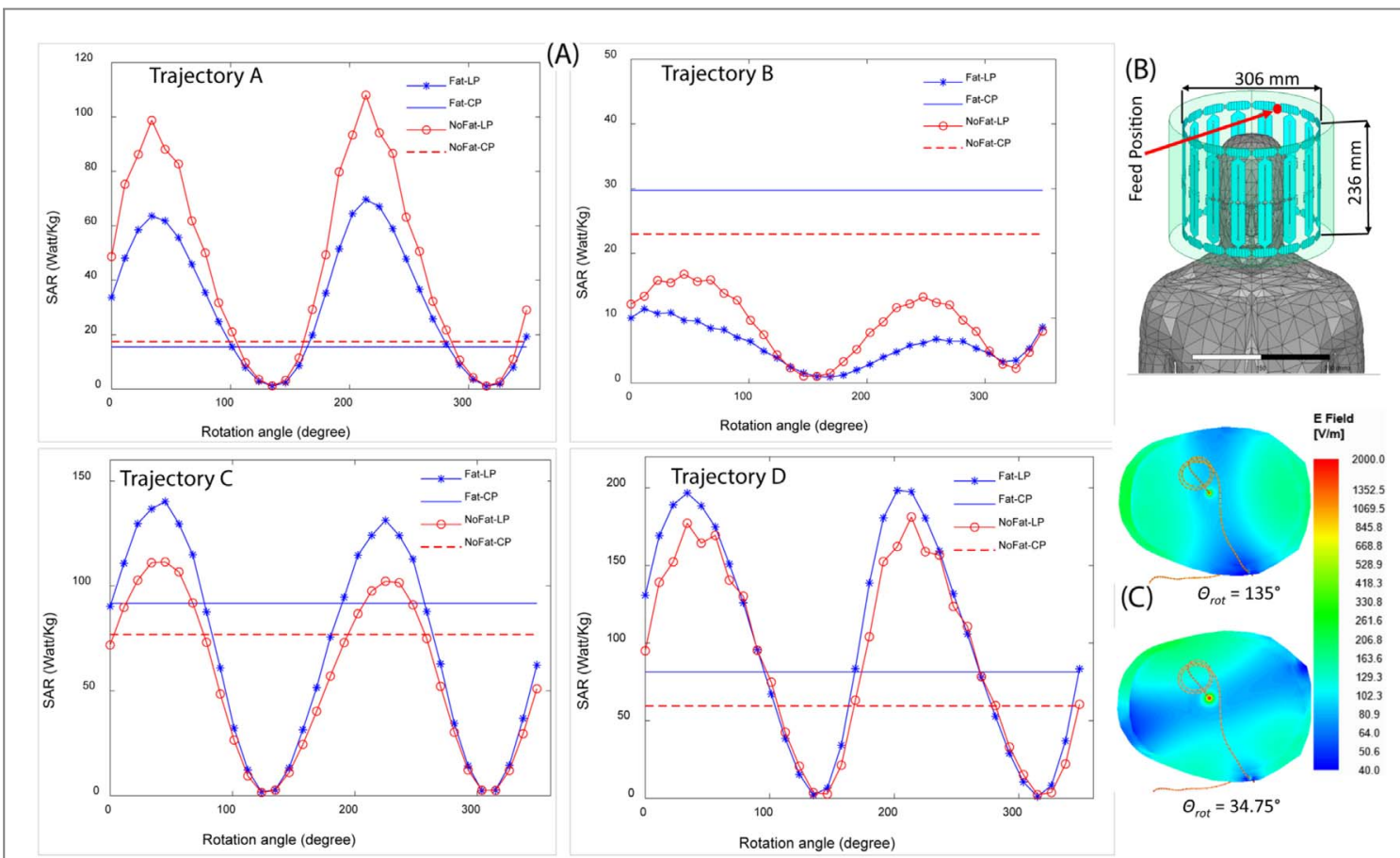

Figure 6. (A) Plot of maximum value of SAR (1g averaged) against angle of rotation of RPA coil for all four different trajectories in the presence and absence of fat tissue. The horizontal lines represent the SAR values generated by the CP body coil. All the SAR values are normalized to $\mathrm{B}_{1}^{+}=2 \mu \mathrm{T}$. (B) RPA coil with body model inserted. The position of feed position corresponding to $\theta=0^{\circ}$ is shown in red. The coil was rotated around patient's head with $11.25^{\circ}$ increments. (C) Complex magnitude of $E$-field on a transverse slice, showing changes in orientation of low $E$-field band with rotation of the RPA coil.

Table 3. SAR reduction efficiencies for different trajectories with and without the chest fat.

\begin{tabular}{lccccc}
\hline \multicolumn{2}{c}{ Trajectories } & & & & \\
& & $\theta_{\text {opt }}$ & $\begin{array}{c}\mathrm{SAR}_{\mathrm{LP}} \\
\left(\mathrm{W} \mathrm{kg}^{-1}\right)\end{array}$ & $\begin{array}{c}\mathrm{SAR}_{\mathrm{CP}} \\
\left(\mathrm{W} \mathrm{kg}^{-1}\right)\end{array}$ & SRE \\
\hline A & Fat & 135 & 1.2 & 15.5 & 92 \\
& No fat & 135 & 1.2 & 17.5 & 93 \\
B & Fat & 157 & 1 & 29.7 & 96 \\
& No fat & 157 & 1 & 23 & 95 \\
C & Fat & 124 & 1.8 & 91.7 & 98 \\
& No fat & 124 & 1.5 & 76.9 & 98 \\
D & Fat & 135 & 2.2 & 81.4 & 97 \\
& No fat & 135 & 3.7 & 59.6 & 93 \\
\hline
\end{tabular}

\subsection{Results}

Figure 6(A) gives the maximum of 1g-averaged SAR at the tip of the lead as a function of RPA coil's angle for different lead trajectories implanted in body models with and without local fat around the IPG. The maximum of $1 \mathrm{~g}$-averaged SAR generated by the CP body coil is also given for comparison. As it can be observed, for all four trajectories the SAR generated by the RPA coil at its optimum rotation angle was significantly lower than the SAR generated by the body coil, irrespective of the presence or absence of fatty tissue. Additionally, the optimum angle for SAR reduction remained relatively unchanged by the inclusion of fatty tissue for all trajectories. Figure 6(C) gives the distribution of $\mathrm{E}$ field for two positions of the RPA coil corresponding to maximum and minimum SAR. SAR values for trajectories A-D for different coil rotation angles with and without fat are given in supplementary file S1 (available online at stacks.iop.org/PMB/66/015008/mmedia).

The SAR reduction efficiencies of the RPA coil for different lead trajectories implanted in the body model with and without fatty tissue are given in table 3 . As the table indicates, the SRE of more than $90 \%$ was achieved using the RPA coil technology compared to the birdcage body coil irrespective of presence of fat.

Finally, we examined the performance of the RPA coil in terms of $\mathrm{B}_{1}^{+}$inhomogeneity by calculating the ratio of the standard deviation and mean of $\mathrm{B}_{1}^{+}$on a transverse plane inside the head located $4 \mathrm{~cm}$ above the coil's isocenter (figure 7(A)) as: 

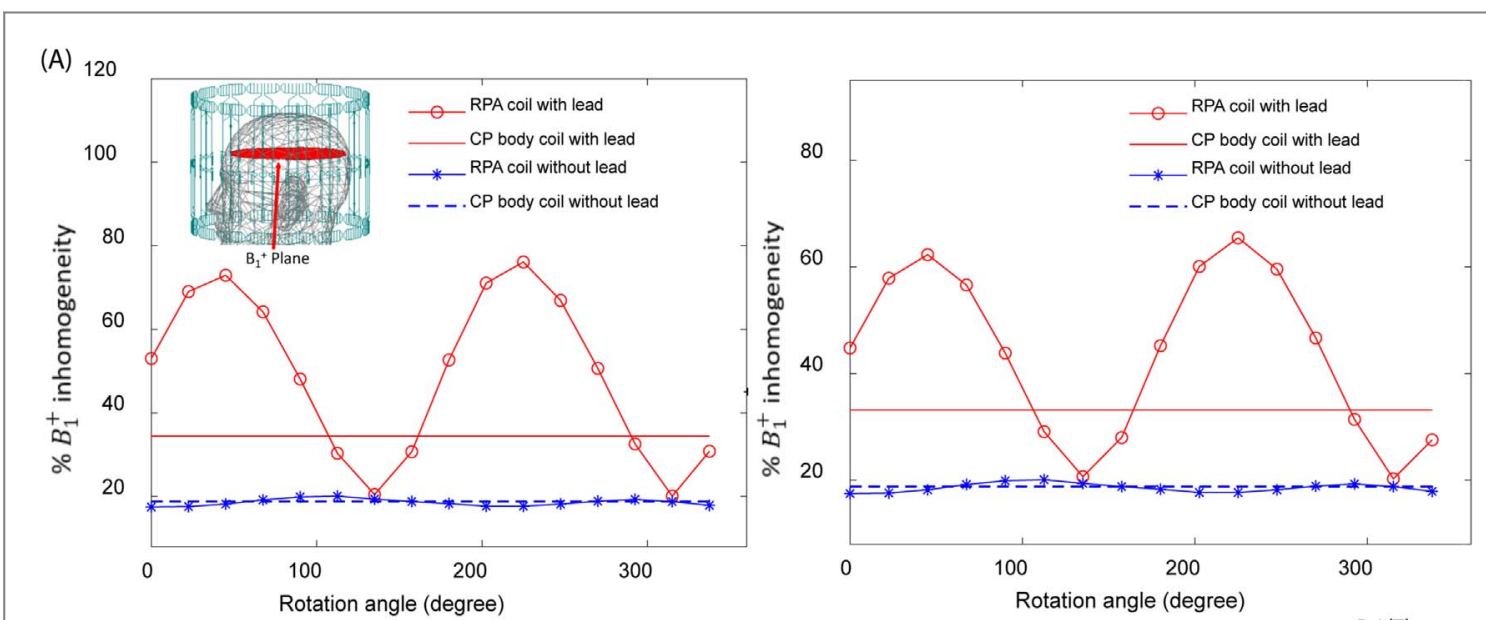

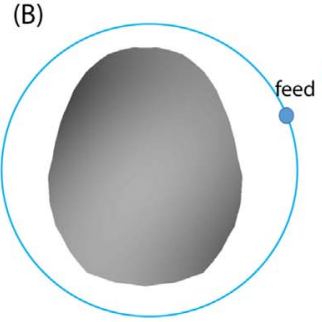

RPA coil, $\Theta_{\text {rot }}=135$ No lead

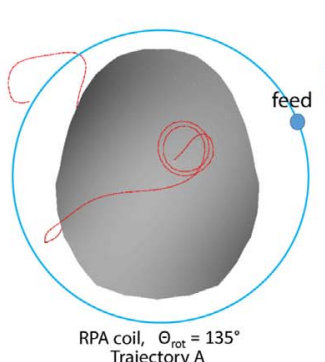

Trajectory A

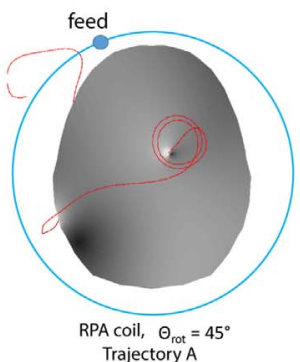

Trajectory A



Trajectory A

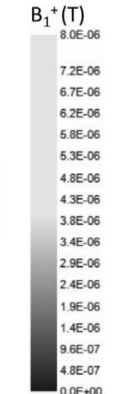

Figure 7. (A) Plot of $\mathrm{B}_{1}^{+}$variation with angle of rotation of RPA coil, in the presence DBS lead (trajectory A) as well as without lead using realistic body model without fat tissue (left) and with fat tissue (right). The corresponding variations for CP body coil are also added as horizontal lines. The transverse plane was covering brain tissue only. (B) Plot of $\mathrm{B}_{1}^{+}$field distribution in the same plane for RPA coil as well as CP body coil. For RPA coil, the plots correspond to minimum $B_{1}^{+}$inhomogeneity $\left(\theta_{\text {rot }}=135^{\circ}\right)$ and maximum $B_{1}^{+}$ inhomogeneity $\left(\theta_{\text {rot }}=45^{\circ}\right)$. The position of feed in each case has been shown by the dot on the circle (figures not to the scale).

$$
\% \mathrm{~B}_{1}^{+} \text {Inhomogeneity }=100 \times \frac{\text { Standard Deviation in } \mathrm{B}_{1}^{+}}{{\text {Mean } \mathrm{B}_{1}^{+}}} .
$$

Figure 7(A) shows the plots of $\mathrm{B}_{1}^{+}$inhomogeneity for lead trajectory $\mathrm{A}$, implanted in the realistic body model with and without fat tissue using both RPA coil and the $\mathrm{CP}$ body coil. Reference values for $\mathrm{B}_{1}^{+}$inhomogeneity in the absence of the implant are also included for comparison. The $\mathrm{B}_{1}^{+}$field of an empty LP birdcage coil is highly uniform, however, this changes when the coil is loaded with a human head (Jin and Chen 1997). Field inhomogeneity inside the head will be specifically affected by the location of the linear feed with respect to the head. From figure 7, the maximum $\mathrm{B}_{1}^{+}$inhomogeneity in the head in the absence of the implant was $20.1 \%$ for the RPA coil (compared to $18.8 \%$ for CP body coil), which occurred when the linear feed was located in front of right ear. The field inhomogeneity was, however, reduced to $17.5 \%$ when the linear feed was located $22.5^{\circ} \mathrm{left}$ of nose.

Presence of metallic implants can worsen the $\mathrm{B}_{1}^{+}$field inhomogeneity, as the induced RF currents along the implant generate secondary magnetic fields, thus distorting the original field distribution (Camacho et al 1995, Lauer et al 2005). Plots in figure 7(A) clearly show that the variation of $\mathrm{B}_{1}^{+}$field inhomogeneity with rotation of RPA coil, in the presence of implant, follows the same trend as the RF heating, with minima and maxima positions coinciding with the SAR minima and maxima (figure 6). Additionally, at optimal position, the $\mathrm{B}_{1}^{+}$field inhomogeneity for RPA coil is reduced below the corresponding level for CP body coil, with as much as $40 \%$ improvement in field inhomogeneity in the phantom without fat and 38\% improvement in the phantom with fat. From figure $7(\mathrm{~B})$ it is observed that $\mathrm{B}_{1}^{+}$field distortion due to presence of implant is minimized when the RPA coil is at its optimal position. For higher heating cases, significant $\mathrm{B}_{1}^{+}$distortion is produced not only by the intracranial part of the DBS lead, but also by the extracranial portions of the lead and extension. The results are consistent with earlier studies involving variation of RF heating and $\mathrm{B}_{1}^{+}$field inhomogeneity/image artifacts (Boutet et al 2019, McElcheran et al 2019).

\section{Discussion and conclusion}

Patients with DBS devices can reap much benefit from MRI if their eligibility for MRI is extended to a wider range of sequences at $1.5 \mathrm{~T}$ as well as to higher field strengths beyond the restrictions imposed by current 
guidelines. As the primary concern for contraindication is risks associated with RF heating, techniques for mitigating such heating can pave the way towards allowing use of 3 T MRI for DBS imaging. Due to high degree of complexity of RF heating phenomena, the results of studies using simplified homogenous models might not provide enough confidence on patient's safety and more contributions incorporating complexity of body tissues are warranted. This study, using experimental measurements as well as numerical simulation, has provided evidence that patient's body composition substantially affects RF heating of DBS devices during MRI. A more important upshot of this work, however, is that it affirms that risk mitigation strategies based on reconfigurable coil technology and DBS surgical lead management are still effective during 3 T MRI, even with variant patient's body compositions.

In the past few years, numerical simulations have been increasingly used to assess safety of medical devices and imaging instruments (Golestanirad et al 2012a, 2012b, Navarro de Lara et al 2020). An important aspect of such practice is to validate simulations against measurements whenever possible, in order to provide confidence in future predictions of such models. Here we show that numerical simulations agree well with experiments in predicting the effect of modification in device configuration as well as patient's body composition on RF heating of DBS implants. Addition of fatty tissue substantially altered the RF heating in simulations, which was in line with what was observed in our experimental setup. Simulations with body models that more realistically resembled human subjects predicted similar trend in variation in RF heating.

The alteration of RF heating at the tip of a DBS implant due to presence of local fat around the IPG might be due to two possible effects. Firstly, the local distribution of incident electric field of MRI scanner will be altered in and in close vicinity of the fat which has low permittivity and conductivity compared to surrounding tissue (Alon et al 2016). This can in turn change the coupling of electric field with the portion of the lead/extension that is inside or close to the fatty tissue. Secondly, the presence of low permittivity fat will change the wavelength of RF fields surrounding the leads which can result in shifts in antenna resonance lengths (Nyenhuis et al 2005, Acikel and Atalar 2011). The substantial alteration of heating patterns due to presence of fat highlights the importance of considering body complexity in RF heating evaluations for implants during MRI.

Another important observation, confirmed with both simulations and measurements, was that lead trajectories with loops positioned at the surgical burr hole, or loops positioned both at the burr hole and toward the temporal bone, maximally reduced the RF heating for both cases with and without inclusion of fat. This suggests that lead management strategies are resilient to variations in patient's body characteristics. It is worthy to note, however, the trajectories with loops placed at the burr hole are easier to implement surgically than the others.

In the context of MRI hardware modification, the performance of the RPA coil technology has not been tested on fully implanted DBS systems using patient models with variant body compositions. Here we showed that when positioned at its optimal angle, RPA coil consistently reduced RF heating compared to the CP body coil for all trajectories irrespective of inclusion or exclusion of fatty tissue. More importantly, the optimal angle for a particular trajectory was relatively unchanged for different body compositions. These finding indicate that effect of patient's characteristics on the optimal rotation angle of the coil is small or negligible, although more study is required to draw definitive conclusions on the degree of coil sensitivity to body characteristics. The RPA coil comes with additional benefit of reducing $\mathrm{B}_{1}^{+}$inhomogeneity which is generated by induced current in implanted leads. When positioned at optimal rotation angle for SAR minimization, the induced currents along the lead are also minimized resulting into concurrent reduction in the $\mathrm{B}_{1}^{+}$field distortion. Such inhomogeneity is the source of the well-known non-susceptibility image artifact around elongated implants (Camacho et al 1995).

Though this study includes tissue heterogeneity by including brain, skull and fat, it does not account for the heterogeneity inside the brain tissue, which can be a subject of further evaluation. Furthermore, representation of fatty tissue in this study has been quite simplified, using either a surface layer or a block of tissue around IPG. Using virtual population models that incorporate a wide distribution of fat can provide additional insight in the future. In this study, the DBS model in the numerical simulations has been represented by a simplified insulated wire with single wire core. However, a head-to-head comparison between simplified versus realistic wire models implanted in heterogeneous body models and routed around different trajectories remains to be done. In addition, the results presented here are based on one model of DBS device (Medtronic, lead 3387, extension 3708660). Further study is required to draw conclusions for a wider range of DBS implant models being used in current practices.

\section{Acknowledgments}

This work was supported by NIH grant R00EB021320. 


\section{ORCID iDs}

Bhumi Bhusal (i) https://orcid.org/0000-0002-4404-9784

Laleh Golestanirad iํ https:// orcid.org/0000-0003-3869-6114

\section{References}

Acikel V and Atalar E 2011 Modeling of radio-frequency induced currents on lead wires during MR imaging using a modified transmission line method Med. Phys. 38 6623-32

Alon L, Deniz C M, Carluccio G, Brown R, Sodickson D K and Collins C M 2016 Effects of anatomical differences on electromagnetic fields, SAR, and temperature change Concepts Magn. Reson. B 46 8-18

Baker K B, Tkach J, Hall J D, Nyenhuis J A, Shellock F G and Rezai A R 2005 Reduction of magnetic resonance imaging-related heating in deep brain stimulation leads using a lead management device Neurosurgery 57 392-7

Bhusal B, Nguyen B T, Sanpitak P P, Vu J, Elahi B, Rosenow J, Nolt M J, Lopez-Rosado R, Pilitsis J, DiMarzio M and Golestanirad L 2020 Effect of device configuration and patient's body composition on the RF heating and nonsusceptibility artifact of deep brain stimulation implants during MRI at 1.5 T and 3T J. Magn. Reson. Imaging (https://doi.org/10.1002/jmri.27346)

Boutet A, Rashid T, Hancu I, Elias G J, Gramer R M, Germann J, Dimarzio M, Li B, Paramanandam V, Prasad S and Ranjan M 2019 Functional MRI safety and artifacts during deep brain stimulation: experience in 102 patients Radiology 293 174-83

Boston Scientific 2019 ImageReady MRI Guidelines for Boston Scientific Deep Brain Stimulation System (https://bostonscientific.com/ content/dam/Manuals/us/current-rev-en/92195369-01_ImageReady ${ }^{\mathrm{TM}}$ A2_MRI_Guidelines_for_Boston_Scientific_Deep_Brain Stimulation_Systems_en-US_s.pdf)

Camacho C R, Plewes D B and Henkelman R M 1995 Nonsusceptibility artifacts due to metallic objects in MR imaging J. Magn. Reson. Imaging 5 75-88

Cheng C-H, Huang H-M, Lin H-L and Chiou S-M 2014 1.5 T versus 3 T MRI for targeting subthalamic nucleus for deep brain stimulation Br. J. Neurosurg. 28 467-70

Eryaman Y et al 2014 Parallel transmit pulse design for patients with deep brain stimulation implants Magn. Reson. Med. 73 1896-903

Feng S, Qiang R, Kainz W and Chen J 2014 A technique to evaluate MRI-induced electric fields at the ends of practical implanted lead IEEE Trans. Microwave Theory Tech. 63 305-13

Fujimoto K, Angelone L M, Lucano E, Rajan S S and Iacono M I 2018 Radio-frequency safety assessment of stents in blood vessels during magnetic resonance imaging Front. Physiol. 91439

Golestanirad L, Angelone L M, Iacono M I, Katnani H, Wald L L and Bonmassar G 2017a Local SAR near deep brain stimulation (DBS) electrodes at 64 and $127 \mathrm{MH}$ z: a simulation study of the effect of extracranial loops Magn. Reson. Med. 78 1558-65

Golestanirad L, Angelone L M, Kirsch J, Downs S, Keil B, Bonmassar G and Wald L L 2019a Reducing RF-induced heating near implanted leads through high-dielectric capacitive bleeding of current (CBLOC) IEEE Trans. Microwave Theory Tech. 67 1265-73

Golestanirad L, Dlala E, Wright G, Mosig J R and Graham S J 2012a Comprehensive analysis of Lenz effect on the artificial heart valves during magnetic resonance imaging Prog. Electromagn. Res.-PIER 128 1-17

Golestanirad L, Iacono M I, Keil B, Angelone L M, Bonmassar G, Fox M D, Herrington T, Adalsteinsson E, LaPierre C and Mareyam A 2017b Construction and modeling of a reconfigurable MRI coil for lowering SAR in patients with deep brain stimulation implants Neuroimage 147 577-88

Golestanirad L, Kazemivalipour E, Keil B, Downs S, Kirsch J, Elahi B, Pilitsis J and Wald L L 2019b Reconfigurable MRI coil technology can substantially reduce RF heating of deep brain stimulation implants: first in-vitro study of RF heating reduction in bilateral DBS leads at 1.5 T PLoS One 14

Golestanirad L, Keil B, Angelone L M, Bonmassar G, Mareyam A and Wald L L 2017c Feasibility of using linearly polarized rotating birdcage transmitters and close-fitting receive arrays in MRI to reduce SAR in the vicinity of deep brain simulation implants Magn. Reson. Med. 77 1701-12

Golestanirad L, Kirsch J, Bonmassar G, Downs S, Elahi B, Martin A, Iacono M-I, Angelone L M, Keil B and Wald L L 2019c RF-induced heating in tissue near bilateral DBS implants during MRI at $1.5 \mathrm{~T}$ and $3 \mathrm{~T}$ : the role of surgical lead management NeuroImage 184 566-76

Golestanirad L et al 2019d Changes in the specific absorption rate (SAR) of radiofrequency energy in patients with retained cardiac leads during MRI at 1.5 T and $3 \mathrm{~T}$ Magn. Reson. Med. 81 653-69

Golestanirad L, Rouhani H, Elahi B, Shahim K, Chen R, Mosig J R, Pollo C and Graham S J 2012b Combined use of transcranial magnetic stimulation and metal electrode implants: a theoretical assessment of safety considerations Phys. Med. Biol. 577813

Guerin B, Angelone L M, Dougherty D and Wald L L 2020 Parallel transmission to reduce absorbed power around deep brain stimulation devices in MRI: impact of number and arrangement of transmit channels Magn. Reson. Med. 83 299-311

Jin J and Chen J 1997 On the SAR and field inhomogeneity of birdcage coils loaded with the human head Magn. Reson. Med. 38953-63

Kalin R and Stanton M S 2005 Current clinical issues for MRI scanning of pacemaker and defibrillator patients Pacing Clin. Electrophysiol. 28 $326-8$

Kazemivalipour E, Keil B, Vali A, Rajan S, Elahi B, Atalar E, Wald L L, Rosenow J, Pilitsis J and Golestanirad L 2019 Reconfigurable MRI technology for low-SAR imaging of deep brain stimulation at 3T: application in bilateral leads, fully-implanted systems, and surgically modified lead trajectories NeuroImage 199 18-29

Kozlov M and Turner R 2009 Fast MRI coil analysis based on 3D electromagnetic and RF circuit co-simulation J. Magn. Reson. 200 147-52

Lattanzi R, Sodickson D K, Grant A K and Zhu Y 2009 Electrodynamic constraints on homogeneity and radiofrequency power deposition in multiple coil excitations Magn. Reson. Med. 61315-34

Lauer U A, Graf H, Berger A, Claussen C D and Schick F 2005 Radio frequency versus susceptibility effects of small conductive implants-a systematic MRI study on aneurysm clips at 1.5 and 3 T Magn. Reson. Imaging 23 563-9

McCabe S and Scott J 2017 A novel implant electrode design safe in the RF Field of MRI scanners IEEE Trans. Microwave Theory Tech. 65 $3541-7$

McElcheran C, Golestanirad L, Iacono M, Wei P-S, Yang B, Anderson K, Bonmassar G and Graham S 2019 Numerical simulations of realistic lead trajectories and an experimental verification support the efficacy of parallel radiofrequency transmission to reduce heating of deep brain stimulation implants during MRI Sci. Rep. 91-14 
McElcheran C E, Yang B, Anderson K J, Golestanirad L and Graham S J 2017 Parallel radiofrequency transmission at 3 Tesla to improve safety in bilateral implanted wires in a heterogeneous model Magn. Reson. Med. 78 2406-15

Medtronic 2015 MRI Guidelines for Medtronic Deep Brain Stimulation Systems (http://mriquestions.com/uploads/3/4/5/7/34572113/ dbs_medtronics_contrib_228155.pdf)

Naehle C P, Zeijlemaker V, Thomas D, Meyer C, Strach K, Fimmers R, Schild H and Sommer T 2009 Evaluation of cumulative effects of MR imaging on pacemaker systems at 1.5 Tesla Pacing Clin. Electrophysiol. 32 1526-35

Navarro de Lara L I, Golestanirad L, Makarov S N, Stockmann J P, Wald L L and Nummenmaa A 2020 Evaluation of RF interactions between a $3 \mathrm{~T}$ birdcage transmit coil and transcranial magnetic stimulation coils using a realistically shaped head phantom Magn. Reson. Med. $841061-75$

Nguyen B T, Pilitsis J and Rad L G 2020 The effect of simulation strategies on prediction of power deposition in the tissue around electronic implants during magnetic resonance imaging Phys. Med. Biol. 65185007

Nordbeck P, Fidler F, Weiss I, Warmuth M, Friedrich M T, Ehses P, Geistert W, Ritter O, Jakob P M and Ladd M E 2008 Spatial distribution of RF-induced E-fields and implant heating in MRI Magn. Reson. Med. $60312-9$

Nyenhuis J, Jallal J, Min X, Sison S and Mouchawar G 2015 Computing in Cardiology Conf. (CinC), 2015) (Piscataway, NJ: IEEE) pp 765-8

Nyenhuis J A, Park S-M, Kamondetdacha R, Amjad A, Shellock F G and Rezai A R 2005 MRI and implanted medical devices: basic interactions with an emphasis on heating IEEE Trans. Device Mater. Reliab. 5467-80

Park S M, Kamondetdacha R and Nyenhuis J A 2007 Calculation of MRI-induced heating of an implanted medical lead wire with an electric field transfer function J. Magn. Reson. Imaging $261278-85$

Serano P, Angelone L M, Katnani H, Eskandar E and Bonmassar G 2015 A novel brain stimulation technology provides compatibility with MRI Sci. Rep. 5

Shrivastava D, Abosch A, Hanson T, Tian J, Gupte A, Iaizzo P A and Vaughan J T 2010 Effect of the extracranial deep brain stimulation lead on radiofrequency heating at 9.4 Tesla (400.2 MHz) J. Magn. Reson. Imaging 32 600-7

Shrivastava D, Abosch A, Hughes J, Goerke U, DelaBarre L, Visaria R, Harel N and Vaughan J T 2012 Heating induced near deep brain stimulation lead electrodes during magnetic resonance imaging with a 3 T transceive volume head coil Phys. Med. Biol. 575651

St Jude Medical 2018 MRI Procedure Information (https://manuals.sjm.com/ /media/manuals/product-manual-pdfs/d/6/d6db9679aaa7-4e22-b1e3-1d8a2ac43b98)

Wintermark M, Albers G W, Alexandrov A V, Alger J R, Bammer R, Baron J-C, Davis S, Demaerschalk B M, Derdeyn C P and Donnan G A 2008 Acute stroke imaging research roadmap Stroke 39 1621-8

Yeung C J, Susil R C and Atalar E 2001 RF safety of wires in interventional MRI: using a safety index Magn. Reson. Med. 47 187-93 